\section{Werlé-Legendre Separation in a Hydraulic Machine Draft Tube}

The three-dimensional turbulent flow in a compact hydraulic machine elbow draft tube is numerically investigated for several operating conditions, covering an extended range around the best efficiency point. Comparisons with the experimental data are presented as validation. The interest is focused on the experimentally observed pressure recovery drop occurring near the best efficiency point. The flow is first analyzed locally by means of a topological analysis, then globally with an energetic approach. The study provides evi-dence for the role played by a Werlé-Legendre separation originating in the bend. The separation is due to the contrasting flow angles imposed by the blades, and the angle resulting from the secondary flow.

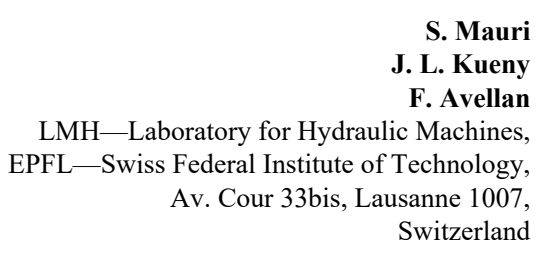

S. Mauri

. L. Kueny

LMH—Laboratory for Hydraulic Machines, Av. Cour 33bis, Lausanne 1007

Switzerland

\section{Introduction}

Draft tubes are components which act to convert a maximum of dynamic pressure into static pressure. A measure of the efficiency of these devices is the static pressure recovery obtained. A large number of hydraulic turbomachinery installations are ageing. Thus, there is potential during refurbishment to implement changes in the design for improved efficiency and associated power output as well as greater operating stability. Usually the runner and guide vanes are focused upon in the refurbishment process. Due to capital construction costs the spiral casing and the draft tube are seldom modified. Unfavorable flow behavior occurs when the runner and the draft tube are unsuitably matched. This can sacrifice flow stability and reduce the optimal operating range of the machine. Typically a sudden drop of the pressure recovery in the draft tube is observed near the best efficiency operating condition. The comprehension of this unexplained phenomenon is the objective of the present study. For this aim an extended range of operating conditions is numerically investigated. After the description of the model, a grid convergence study is presented, then comparisons with experimental data are briefly shown as validation. The flow is first analyzed globally with an energetic approach, then locally by means of a topological analysis. The framework introduced by Tobak and Peake [1] is adopted to describe the topological evolution of the flow in the draft tube as the flow rate is varied. A debate on the terminology can be found in Hornung and Perry [2] and other references cited therein.

\section{Case}

Experimental studies are carried out on a vertical axis reduced scale model (1:10) of an existing water turbine at the test rig facilities of the EPFL-Laboratory for Hydraulic Machines. A high specific speed Francis runner supplies the symmetrical draft tube with a single pier (Fig. 1). The numerical flow analysis is carried out at a constant head for 14 flow rates ranging from $90 \%$ to $110 \%$ of the best efficiency discharge. The Reynolds number based on the inlet mean velocity and diameter is $\operatorname{Re}_{D} \sim 1.510^{6}$. The inlet conditions are experimentally investigated on the symmetry axis diameter at six operating points by means of the local density approximation (LDA) technique. The three components of the velocity and the turbulent kinetic energy profile (Fig. 2) are obtained through two positions of a two-dimensional (2D) laser probe, [4]. The measurement uncertainties are estimated to be less than $3 \%$. The other conditions are linearly interpolated from the measurements.
Modeling. Three-dimensional (3D) steady Reynoldsaveraged Navier-Stokes (RANS) flow computations with the standard $k-\epsilon$ turbulence model and logarithmic wall functions are performed. The flow in the draft tube is simulated using the commercial code CFX-TASCFLOW 2.10. Details on the code are given in Refs. [5,6]. The computation is considered converged to the steady solution when the value of the maximal normalized equation residual is less than $10^{-4}$. The fluid properties are set to the values corresponding to the water at atmospheric conditions. The calibration of modeling parameters has been performed in previous studies, see Refs. [7,8], and is here briefly summarized. The inlet profile is specified at the inlet using a cubic spline. A linear interpolation is applied to the circumference. The radial component is imposed as $C_{r}=C_{r} \tan (\theta r / R)$. The inclination of the velocity vector in the radial direction is, therefore, determined by the geometry of the cone. This theoretical profile performs better than the profile extrapolated from the relatively few and wiggly measured values. The influence of the radial component is important in spite of its small magnitude. The nearest measurement point is at 0.076 inlet diameter from the wall. The extrapolation of the velocity profile from the last measurement point to the wall has a strong influence on the flow prediction in the draft tube. The influence of the momentum thickness on the efficiency of a diffusor is well known. The velocity components at the wall are set as a factor of the velocity at the nearby interior measurement point. Best results with respect to measurements are achieved with a factor of 0.9 . The standard $k-\epsilon$ model assumes that $\epsilon=k^{3 / 2} / L_{\epsilon}$. For the determination of $\epsilon$ from the measured turbulent kinetic energy, the length scale $L_{\epsilon}$ defining the size of the largest eddies must be specified. This parameter has an important influence on the overall flow. $L_{\epsilon}=0.002 \mathrm{D}$ leads to the best results. Several models of the outlet region show a limited influence on the upstream flow. The domain is, therefore, simply prolonged with a downstream channel of length $2 \mathrm{D}$. At the outlet the flow is considered developed. Several two-equation turbulence models and one second-order closure scheme have been tested. While the twoequation turbulence models perform similarly, the Reynolds-stress model does not increase agreement with the experimental results in spite of the theoretically greater potential. A structured multiblock mesh discretizes the geometry of the draft tube model. A butterfly topology with a C-shaped grid around the pier is used. The $y^{+}$values of the first grid points lie in the validity range of the model.

Verification. An a posteriori numerical error estimation based on the generalized Richardson extrapolation not requiring any restriction to integer refinement and applicable to solution functionals is carried out. Following Roache [10] the more conservative Grid Convergence Index is also reported. The flow rate in the left channel obtained with three meshes is compared at $\varphi^{*}=0.994$ in 


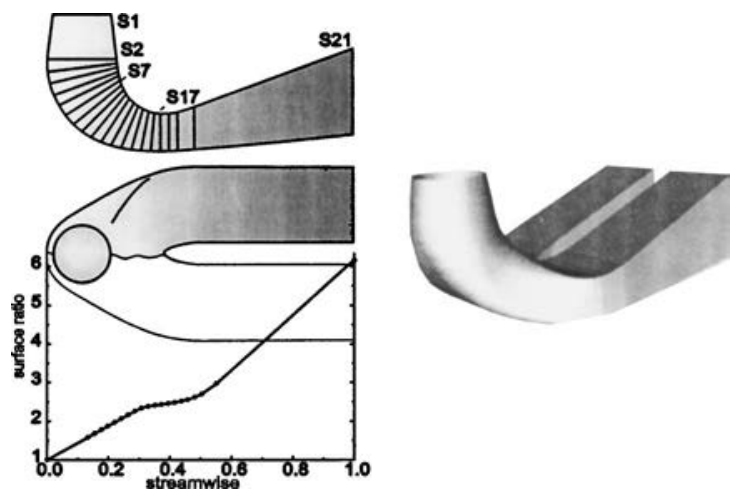

Fig. 1 Investigated geometry. Cross area evolution. Sections definition.

Table 1 . The number of nodes corresponds to the effective number of points in the draft tube geometry (double nodes at the block interfaces are counted only once, the downstream channel is not considered). The grid refinement is reported simply in terms of total number of grid points used in the two meshes as $r_{i j}$ $=\left(N_{i} / N_{j}\right)^{1 / 3}$. The meshes have the same topology but the grid refinement is not uniform in the space. This may give an inaccu-
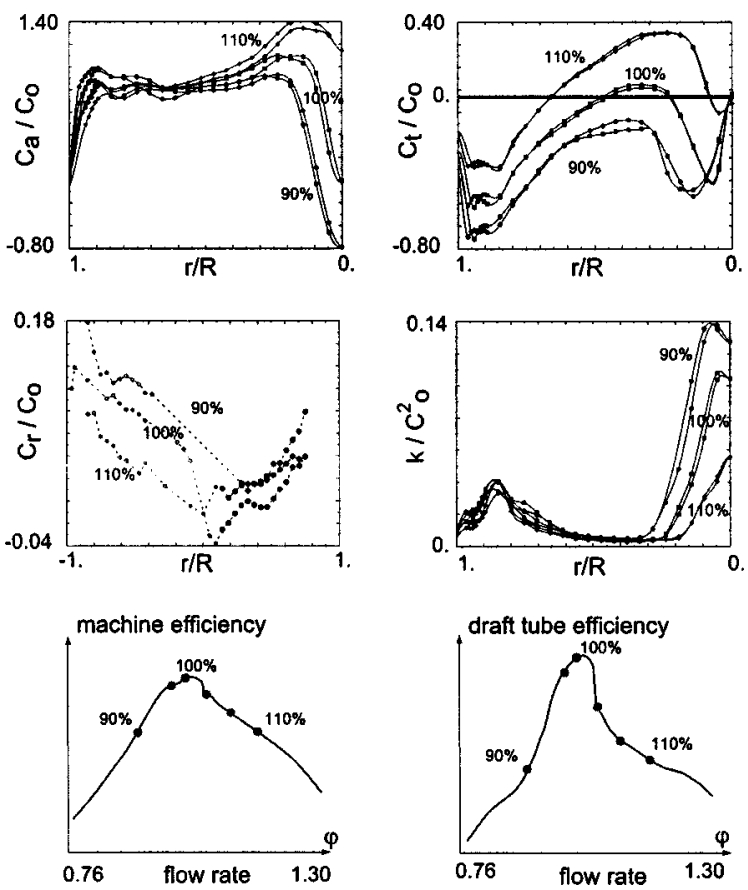

Fig. 2 Measured velocity and kinetic energy inlet profiles (filled points correspond to the external radius). $C_{a}$ : Axial velocity component, $C_{t}$ : Circumferential velocity component, $C_{r}$ : Radial velocity component, $\boldsymbol{k}$ : Turbulent kinetic energy, $C_{0}$ : Mean sectional velocity magnitude. Machine and draft tube efficiency with the main operating points.

Table $1 \varphi^{*}=0.994$. N: Number of nodes, I: Flow rate in the left channel [\%], E: Estimated fractional error, GCI: grid convergence index. Values for $E, G C l$ are reported in [\%] using $p$ $=2$ as order of the discretization.

\begin{tabular}{lcc}
\hline \hline$N_{1}=1,107,237$ & $N_{2}=633,720$ & $N_{3}=328,360$ \\
$l_{1}=63.232$ & $l_{2}=63.309$ & $l_{3}=63.445$ \\
$E_{12}=0.27$ & $E_{12}=0.39$ & $E_{23}=0.60$ \\
$G C I_{12}=0.81$ & $G C I_{3}=1.17$ & $G C I_{23}=1.82$ \\
\hline \hline
\end{tabular}

Table 2 (a) $\varphi^{*}=0.919$ (b) $\varphi^{*}=1.108$. See caption in Table 1.

\begin{tabular}{|c|c|c|}
\hline $\begin{array}{l}l_{1}=72.526 \\
E_{12}=0.06 \\
G C I_{12}=0.17\end{array}$ & $\begin{array}{c}(a) \\
l_{2}=72.544 \\
E_{12}=0.08 \\
G C I_{12}=0.24\end{array}$ & $\begin{array}{c}l_{3}=72.576 \\
E_{23}=0.12 \\
G C I_{23}=0.37\end{array}$ \\
\hline $\begin{array}{l}l_{1}=65.094 \\
E_{12}=0.93 \\
G C I_{12}=2.80\end{array}$ & $\begin{array}{c}l_{2}=65.367 \\
E_{12}=1.44 \\
G C I_{12}=4.06\end{array}$ & $\begin{array}{c}l_{3}=65.886 \\
E_{23}=2.23 \\
G C I_{23}=6.71\end{array}$ \\
\hline
\end{tabular}

rate estimation of the accuracy depending on whether the grid refinement occurs in the critical areas or not. Depending on the mesh resolution at the inlet the resulting flow rate will change and must be corrected to retrieve the measured value by multiplying the velocity field by a factor. The slight differences in the inlet boundary conditions introduce an additional inaccuracy. The meshes seem to be in the asymptotic range; the actual asymptotic rate of convergence is indeed $p=1.98$, to be compared with the theoretical order $p=2$. These results are expected to be only partially representative for other operating conditions due to the important flow differences. The results at the points $\varphi^{*}=0.919-1.108$ are compared in Table 2. The mesh with $N_{3}$ $=328,360$ nodes insures a GCI smaller than $7 \%$ for all operating points. The results obtained with these meshes show the same flow topology and compare very similar with respect to the measured velocity and pressure profiles on several sections. The coarser mesh is, therefore, adequate for our purposes.

\section{Validation}

The comparison with measurements is not emphasized here, the objective being the characterization of the "numerical flow." Only the recovery factor and the velocity field at the draft tube outlet are shown. Several other comparisons on the whole geometry have been performed showing that while the main flow features and trends are correctly captured, locally important differences occur, see, Refs. [7-9]. The recovery factor is compared with the measurements in Fig. 3. The global behavior is fairly well predicted. The pressure recovery drop takes place at the same flow rate observed experimentally, however, the computations overestimate the recovery factor over the whole range, with a maximal difference of $14 \%$ of the measured value. Near the best efficiency conditions the maximal difference is $6 \%$. The velocity field at the draft tube outlet is compared for the two extreme operating points in Fig. 4.

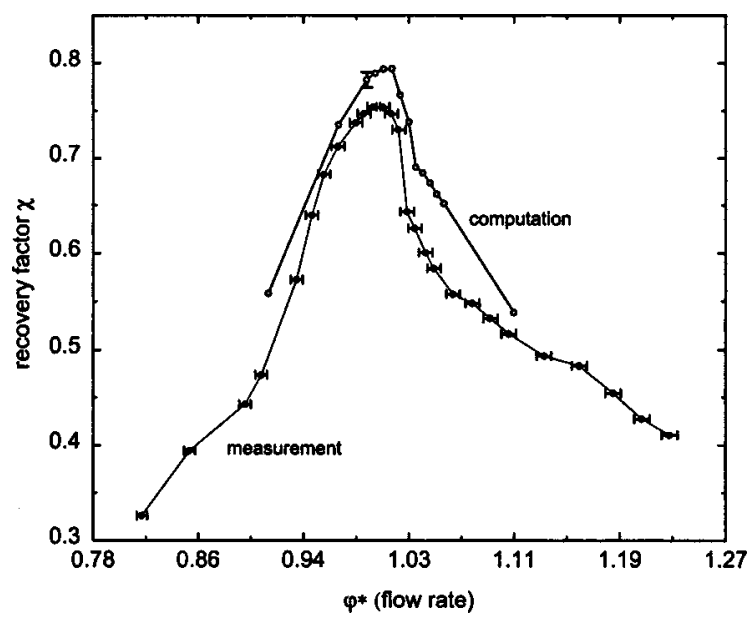

Fig. 3 Static pressure recovery. Comparison measurementcomputation. The $\mathrm{GCl}$ is reported for $\varphi^{*}=0.994$. 


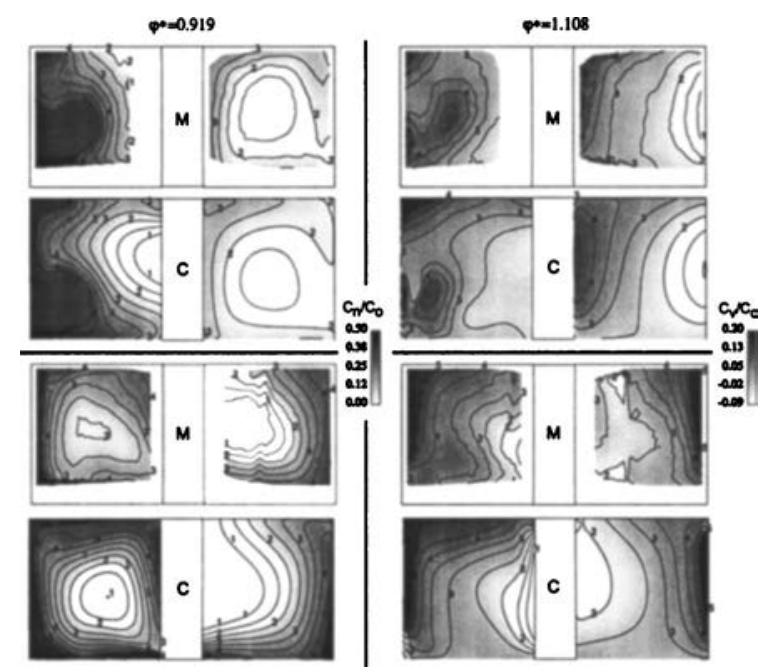

Fig. 4 Comparison measurements $(M)$-computation $(C)$ at the draft tube outlet. Normal $\left(C_{n}\right)$ and vertical $\left(C_{v}\right)$ velocity components, $C_{0}$ : mean sectional velocity magnitude. $\varphi^{*}=0.919$ and $\varphi^{*}=1.108$. The measurements do not cover the whole channels' surface.

Static Pressure Recovery. The specific static pressure energy recovery is summarized in Fig. 5 over the whole computed operating range. Most of the recovery occurs in the cone. The efficiency drop taking place in the second half of the bend is clearly visible in the range $\varphi^{*}=1.021-1.049$. This deficiency is partially recovered in the last part. The domain is split into two symmetrical parts allowing a comparison between the left and the right
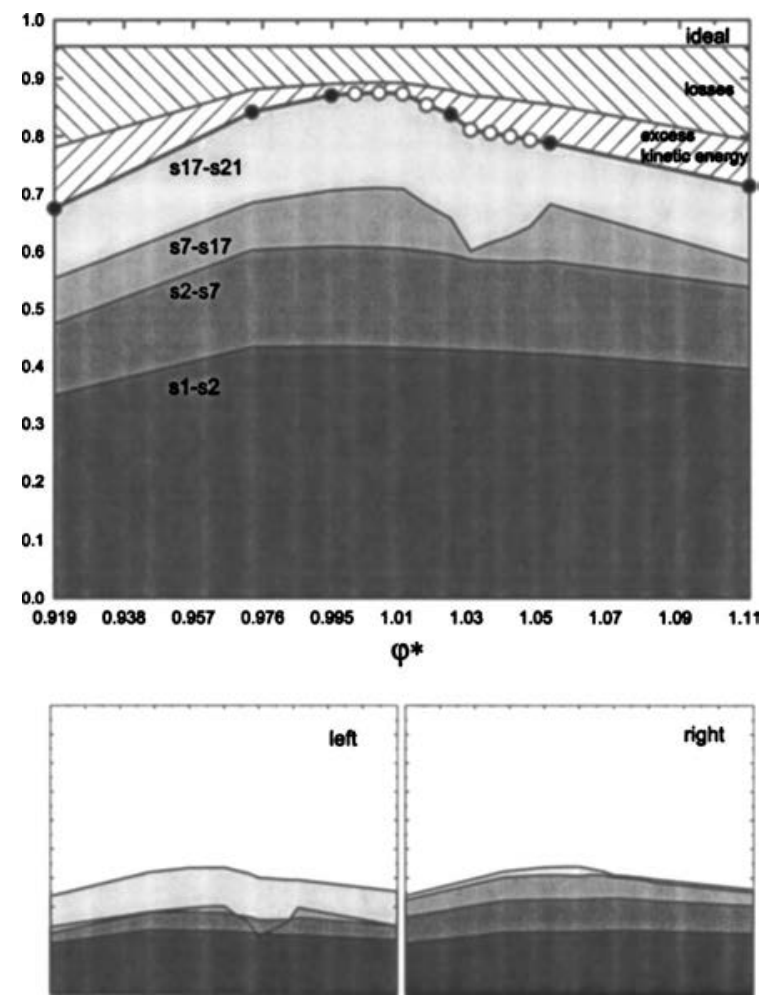

Fig. 5 Computed local mean specific static pressure coefficient. The recovery occurring in the cone (s1-s2), first half of the bend (s2-s7), second half of the bend (s7-s17) and diffuser (s17-s21) and in the left- and right-side of the draft tube are distinguished.

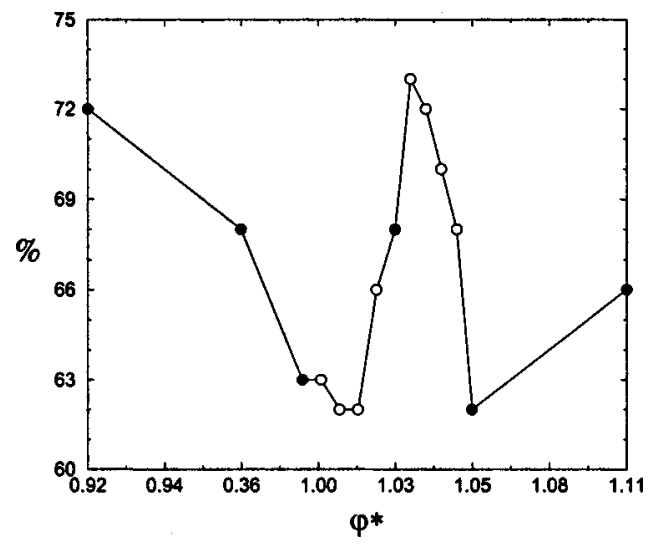

Fig. 6 Predicted flow rate percentage in the left channel over the operating range

channel, even upstream at the pier's leading edge. The recovery drops on the left side due to a flow acceleration, while on the right channel the pressure recovery is very small in the straight diffuser, indicating an unfavorable flow situation. The flow rate distribution in the channels is shown in Fig. 6. The right channel is clearly blocked in the efficiency drop region.

Flow Topology. The main characteristics of the skin friction lines are investigated. Experimentally the flow patterns at the wall can be obtained by means of the oil film method. The power of topological visualization is that, given the singular (critical) points and their principal tangent curves, an observer can visually infer the shape of other tangent curves and hence the structure of the whole vector field. A representation of the global topology is much more readily visualized than the original data set. The main flow characteristics are summarized in Fig. 7, where the change of the flow direction in the right channel due to the increase of the secondary flow introduced by the bend, and to the change of the rotation direction at the inlet (Fig. 2), is put in evidence by means of separation lines. These are lines drawn in the flow toward which other trajectories are asymptotic. A universal definition of separation in a three-dimensional flow is still subject of debate. The specification of separation by means of a reverse flow or vanishing wall shear stress is usually inadequate in threedimensional flows. A necessary condition for the occurrence of flow separation is the convergence of skin friction lines onto a separation line. The regions of flow separation are important because of the reduced kinetic energy and the consequent blockage effect that these regions can introduce. The point before the efficiency drop shows local (not passing through any critical point) separation lines at the surface. After the drop the flow stemming from the cone region situated at the outer side with respect to the
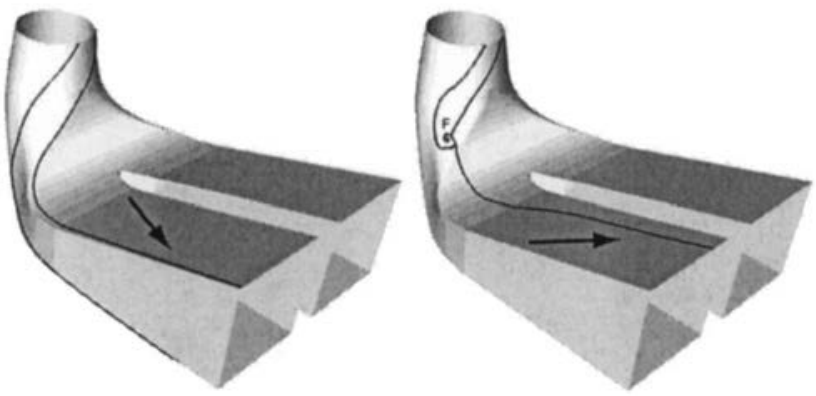

Fig. 7 Predicted separation lines and flow direction in the right channel before $\left(\varphi^{*}=0.994\right)$ and after $\left(\varphi^{*}=1.021\right)$ the efficiency drop 


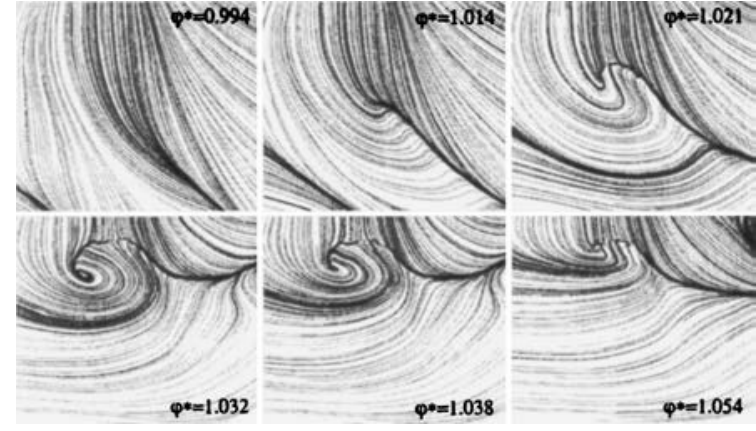

Fig. 8 Computed skin friction lines, onset on the surface of a focus in company with a saddle point. Before the efficiency drop: $\varphi^{*}=0.994, \varphi^{*}=1.014$, after: $\varphi^{*}=1.021, \varphi^{*}=1.032$, $\varphi^{*}=1.038, \varphi^{*}=1.054$.

bend curvature is forced by the secondary flow to the inner side, while the flow angle imposed by the blade geometry remains constant for all operating points. The collision of these two flow directions leads to the onset on the surface of a focus in company with a saddle point leading to a global Werlé-Legendre separation (see also Fig. 8, showing the wall region where the onset of the critical points occurs). In this particular form of separation one leg of the line of separation emanating from the saddle point winds into the focus to form the curve on the surface from which the dividing surface stems. The focus on the wall extends into the fluid as a concentrated vortex filament, while the surface rolls up around the filament. This flow behavior was first hypothesized by Legendre in 1965 and confirmed by the experiments of Werlé (1962). The cooperation between these two scientist is described in Ref. [3]. As the flow rate reaches a critical value the flow bifurcates, breaking the symmetry of the precedent flow and adopting a form of lesser symmetry in which dissipative structures arise to absorb just the amount of excess available energy that the more symmetrical flow no longer was able to absorb. The flow pattern in the inner domain can be seen in Fig. 9, where the vortex core originating from the focus and the tangential streamlines on two cross-sections are shown. There is a practical difficulty to define the separation region delimited by the stream surface originating from the line of separation. A simple separation region extraction algorithm is used to define a volume. Streamlines are started from the zone at the wall where separation occurs. A streamline is then equidistantly divided and from each point the intersection of the corresponding normal plane with all other streamlines is computed. From each group of points the mean point is calculated and used to define a new line and the procedure is repeated until the desired convergence is achieved. From each cloud of points, corresponding to the intersection of the normal plane of this mean line with all streamlines, the best fitting ellipse to the external points of the cloud is computed. The resulting tube

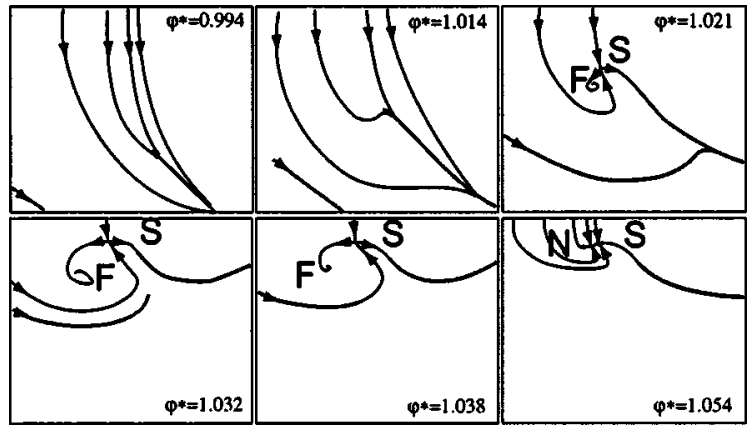

Fig. 9 Computed vortex core and separation line, $\varphi^{*}=1.021$

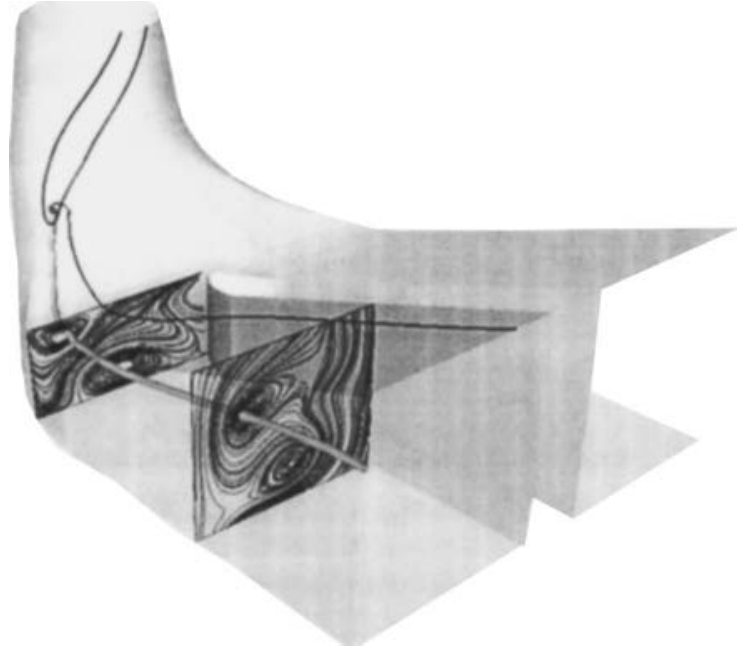

Fig. 10 Predicted vortex evolution. Before the drop: $\varphi^{*}=1.014$, after: $\varphi^{*}=1.021, \varphi^{*}=1.027, \varphi^{*}=1.032, \varphi^{*}=1.038$. The last picture summarizes the first four operating conditions (lighter core: $\varphi^{*}=1.014$, darker core: $\varphi^{*}=1.032$ ).
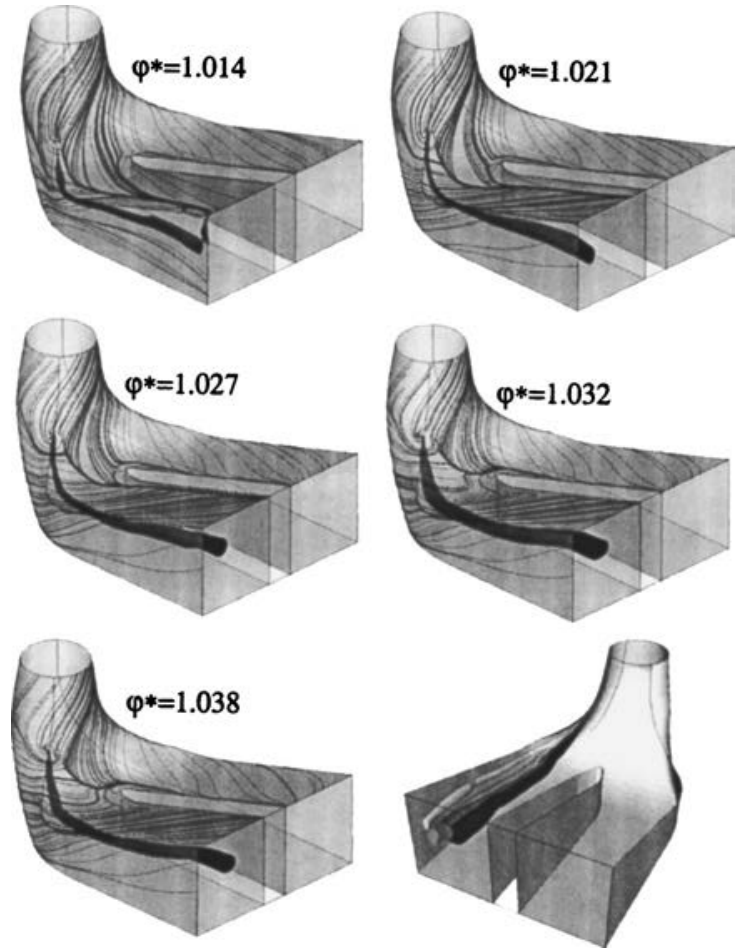

Fig. 11 Interpretation of Fig. 8. S: Saddle, F: Focus, N: Node.

with elliptic sections approximates the separation zone. In this case the separation forms a swirling structure. The vortex evolution is shown in Fig. 10. Just prior to the drop the separated region is small and attached to the sidewall. Then the vortex structure is formed and moves toward the center of the section and increases its size and strength until $\varphi^{*}=1.032$, then begins to decay and at $\varphi^{*}=1.054$ the focus disappears (Fig. 11). The relationship to the flow rate distribution shown in Fig. 6 and to the pressure recovery illustrated in Fig. 5 is evident.

\section{Conclusion}

The origin of the static pressure recovery drop can be clearly localized in the bend region by means of an energetic analysis. 
The study of the skin friction lines in this region shows the onset of a global Werlé-Legendre separation as the flow rate reaches a critical value. The topological structure of the skin friction field bifurcates with the emergence of a saddle point and a focus in the surface pattern. The evolution of the vortex region originating in the focus is visualized in the inner flow clearly illustrating how the separation blocks the right channel leading to a flow acceleration in the other channel. The static pressure recovery is strongly affected, leading to an important loss in the overall machine efficiency.

\section{Acknowledgments}

This study is established within the Flindt project (Eureka No. 1625) whose participants are EPFL, Electricite de France, Alstom Power, General Electric Canada, VA Tech Escher Wyss, and Voith Hydro who are gratefully acknowledged. The project is also supported by the Swiss Federal Commission for Technology and Innovation (PSEL) and the German Ministry of Science and Technology (BMBF). The authors wish to thank G. Ciocan and J. Arpe for the experimental data and the staff of the Laboratory for Hydraulic Machines for providing support in the experimental and numerical work.

\section{Nomenclature}

$$
\begin{aligned}
A_{\text {ref }} & =\text { Reference section area } \\
C & =\text { Velocity field } \\
C_{0} & =\text { Mean local normal velocity } \\
D=0.4[\mathrm{~m}] & =\text { Runner outlet diameter } \\
E_{k_{o}}=\left\langle C^{2} / 2\right\rangle_{\mathrm{Q}} & =\text { Inlet mean kinetic specific energy } \\
e_{p_{i}}=E_{p_{i}} / E_{k_{o}} & =\text { Local mean specific static pressure energy } \\
E_{p_{i}}=\langle p / \rho\rangle_{\mathrm{Q}_{\mathrm{i}}} & =\text { coefficient } \\
\epsilon & =\text { Tocal mean static pressure specific energy } \\
k & =\text { Turbulent kinetic energy } \\
L_{\epsilon} & =\text { Turbulent eddy length scale } \\
Q & =\text { Flow rate } \\
r & =\text { Radius } \\
R & =\text { Inlet radius } \\
\operatorname{Re}_{D}=C_{0} D / v & =\text { Reynolds number }
\end{aligned}
$$

$$
\begin{aligned}
y^{+} & =\text {Nondimensional distance from the wall } \\
\chi=\left((1 / \rho) \Delta P_{d t}\right) / & \\
\left(0.5\left(Q / A_{\text {ref }}\right)^{2}\right) & =\text { Pressure recovery factor } \\
\Delta P_{d t}= & \text { Mean wall pressure difference between } \\
v & \text { draft tube inlet and outlet } \\
\varphi & =\text { Water kinematic viscosity } \\
\varphi^{*} & =\text { Flow rate coefficient } \\
\rho & =\text { the best efficiency point } \\
\theta & =\text { Haler density } \\
\langle f\rangle_{Q}=(1 / Q) & \\
\times \int_{A} f \vec{c} . \vec{n} d A & =\text { Volume opening angle }
\end{aligned}
$$

\section{References}

[1] Tobak, M., and Peake, D. J., 1982, "Topology of Three-Dimensional Separated Flows," Annu. Rev. Fluid Mech., 14, pp. 61-85.

[2] Hornung, H., and Perry, A. E., 1984, "Some Aspects of Three-Dimensional Separation, Part I: Streamsurface Bifurcations," Z. Flugwiss. Weltraumforsch., 8, Heft 2, pp. 77-87.

[3] Délery, J. M., 2001, "Toward the Elucidation of Three-Dimensional Separation," Annu. Rev. Fluid Mech., 33, pp. 129-54.

[4] Ciocan, G. D., Kueny, J.-L., and Avellan, F., 2000, “Optical Measurement Techniques for Experimental Analysis of Hydraulic Turbines Rotor-Stator Interaction," Proceedings of 2000 ASME Fluids Engineering Conference, Boston, June 11-15.

[5] Raw, M. J., Galpin, P. F., and Hutchinson, B. R., 1989, “A Colocated Finite Volume Method for Solving the Navier-Stokes Equations for Incompressible and Compressible Flows in Turbomachinery: Results and Applications," Proceedings Thirty-Sixth Annual General Meeting Canadian Aeronautics and Space Institute, Ottawa Ontario, III.

[6] AEA Technology Engineering Software Limited, 2000, "CFX-TASCflow Theory Documentation Version 2.10," Waterloo, Ontario, Canada N2L 5Z4.

[7] Mauri, S., Kueny, J.-L., and Avellan, F., 2000, "Numerical Prediction of the Flow in a Turbine Draft Tube-Influence of the Boundary Conditions," Proceedings of 2000 ASME Fluids Engineering Conference, Boston, June 11-15.

[8] Mauri, S., 2002, "Numerical Simulation and Flow Analysis of an Elbow Diffuser," Ph.D. thesis EPFL $N^{\circ} 2527$, Lausanne, http://lmhwww.epfl.ch/ Publications/Theses/Mauri/MauriPhD.html

[9] Mauri, S., Kueny, J.-L., and Avellan, F., "Flow Simulation in an Elbow Diffuser: Verification and Validation," Proceedings of the 21st IAHR Symposium on Hydraulic Machinery and Systems, Lausanne, Switzerland, 9-12 September, 2002, pp. 231-240.

[10] Roache, P. J., 1997, "Quantification of Uncertainty in Computational Fluid Dynamics,” Annu. Rev. Fluid Mech., 29, pp. 123-160. 